Chirurg 2005 · 76:270-272

DOI 10.1007/s00104-004-0985-7

Online publiziert: 25. Januar 2005

(c) Springer Medizin Verlag 2005

\author{
B. Hoksch - T. Weber · M. Beshay - R. Stein · J. Schardt - R. A. Schmid \\ DMLL - Klinik für Thoraxchirurgie, Universitätsspital Bern
}

\title{
Differentialdiagnose des pulmonalen Infiltrates nach Pneumothorax-Drainage
}

\section{Fallbericht}

Anamnese. Die 46-jährige Patientin stellte sich in unserer Notfallambulanz mit seit einer Woche bestehendem Hustenreiz und Dyspnoe vor.

Befunde. Fünf Tage zuvor war vom Hausarzt eine Thoraxröntgenaufnahme vorgenommen worden, welche bereits einen Pneumothorax rechts von ca. $4 \mathrm{~cm}$ Breite nachwies. Eine Drainageneinlage oder Punktion des Pneumothorax war zum damaligen Zeitpunkt nicht erfolgt. In der aktuellen Röntgenaufnahme sah man jetzt einen Totalkollaps der rechten Lunge ohne Mediastinalverschiebung.

Therapie. Unmittelbar nach Einlage einer Thoraxdrainage in Bülau-Technik und Anlage von $-20 \mathrm{~cm} \mathrm{H}_{2} \mathrm{O}$-Sog klagte die Patientin über eine Zunahme des Hustenreizes mit teilweise schaumigem Auswurf. Weiterhin entwickelte die Patientin eine leichte Hypoxämie. Die Computertomographie zeigte ein unilaterales breitflächiges Infiltrat rechts (• Abb. 1, 2).

\section{Diagnose: \\ Reexpansionsödem der Lunge}

Bei afebriler und laborchemisch (Leukozyten, CrP) unauffälliger Konstellation ohne anamnestische Hinweise auf stattgehabte oder aktuelle Infektion der Luftwege musste die primäre Verdachtsdiagnose einer Pneumonie verlassen werden. Das breitflächige Infiltrat wurde dann als ein Reexpansionsödem identifiziert.

Verlauf. Da die Patientin keine weiteren schwerwiegenden respiratorischen Einschränkungen entwickelte, konnte auf eine Respiratortherapie verzichtet und eine symptomatische Therapie eingeleitet werden. Das Kontroll-CT $48 \mathrm{~h}$ später wies eine Regredienz des Ödems nach (• Abb. 3). Als Ursache des Pneumothorax konnte im CT eine bullöse Veränderung im Mittellappen ausgemacht werden. Diese wurde dann nach Stabilisierung des Allgemeinzustandes der Patientin thorakoskopisch reseziert und eine Pleurabrasio durchgeführt (• Abb. 4). Die Patientin verließ bei weiterem unauffälligen Verlauf am 6. postoperativen Tag die Klinik.

\section{Diskussion}

Das Reexpansionsödem, 1958 erstmalig von Carlson beschrieben, tritt v. a. bei einem „chronischen“, ausgedehnten Pneumothorax oder Pleuraerguss auf, wird aber auch bei einem kleinen Pneumothorax, der erst kurzzeitig besteht, beschrieben. Critchley et al. berichten weiterhin über ein Reexpansionsödem im Rahmen der thorakoskopischen Evakuation eines Pleuraergusses, Scalzetti et al. beobachteten diese Komplikation nach Talkpleurodese $[2,6,7]$. Ein Reexpansionsödem nach High-frequency-jet-Ventilation wird von Fujino et al. beschrieben [3].

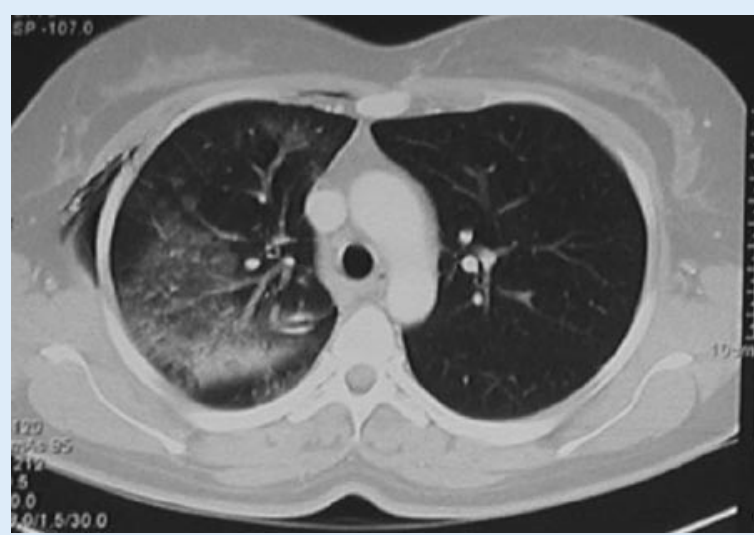

Abb. $1 \Delta$ Breitflächiges Infiltrat der rechten Lunge

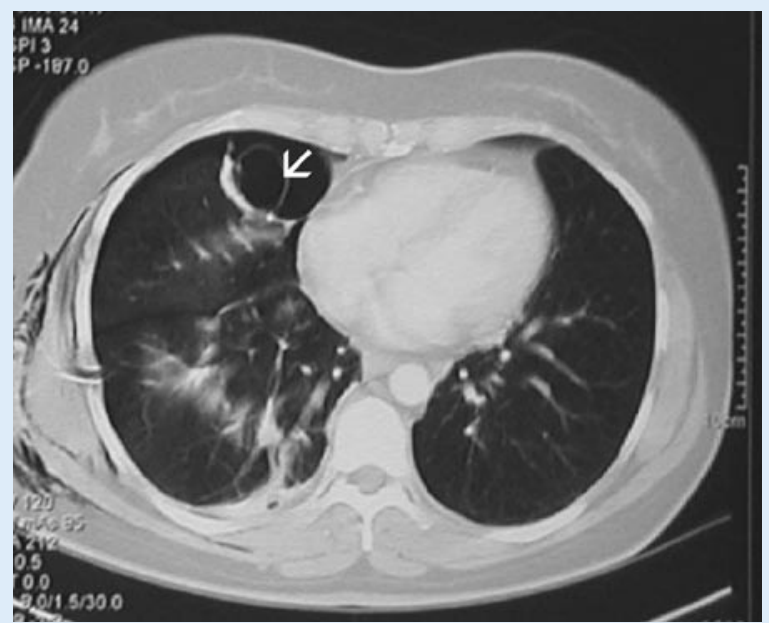

Abb. $2 \Delta$ Große bullöse Veränderung im Mittellappen (Pfeil) 
Zur Pathogenese des Reexpansionsödems werden parallel zu Untersuchungen beim ARDS, Postpneumonektomieödem und Lungentransplantation u. a. eine gesteigerte vaskuläre Permeabilität der hypoxisch geschädigten Lunge und ein Surfactant-Verlust diskutiert $[8 ; 9]$.

In tierexperimentellen Studien wurde die Rolle von biochemischen Mediatoren untersucht. Dabei konnte gezeigt werden, dass insbesondere IL-8 und MCP1 eine entscheidende Rolle bei der Entwicklung des Reexpansionsödems zu spielen scheinen [5]. Im Jahr 2002 gelang Suzuki et al. der Zytokinnachweis in der Ödemflüssigkeit der Lunge [9]. Weiterhin steigern aktivierte freie Radikale die mikrovaskuläre Permeabilität im Rahmen der Lipidperoxidationskaskade [8].

Die Klinik des Reexpansionsödems kann vom asymptomatischen Zufallsbefund bis hin zu schwerwiegenden kardiorespiratorischen Einschränkungen mit der Notwendigkeit zur kurzzeitigen Respiratortherapie reichen. Entsprechend ist die Therapie dem klinischen Bild anzupassen, wobei eine CPAP-Beatmung bei respiratorischer Insuffizienz favorisiert wird [4]. Die Symptomatik (Husten, schaumiger Auswurf, Dyspnoe bis hin zur Ateminsuffizienz) kann dabei entweder sofort, d. h. unmittelbar nach der Reexpansion der Lunge, oder mit einer Verzögerung von bis zu $24 \mathrm{~h}$ einsetzen. In der Regel sind die Befunde - wie auch im Fall unserer Patientin - innerhalb von 48-72 h regredient. Klinisch sowie radiologisch lässt sich ein akuter Pneumothorax im Sinne eines Spannungspneumothorax sicher abgrenzen. Die ödematösen Veränderungen, welche oft fälschlicherweise als entzündliche Infiltrate fehlgedeutet werden, treten in der Regel ipsilateral auf. In einer Sammelstatistik von Mahfood et al. wird die Gesamtmortalitätsrate beim Reexpansionsödem mit 19\% (9 von insgesamt $47 \mathrm{~Pa}$ tienten) angegeben [4]. Patienten mit einem Reexpansionsödem sollten einer entsprechenden kardiopulmonalen Überwachung zugeführt werden.

Generell, aber insbesondere bei einem länger bestehenden Pneumothorax oder einem ausgedehnten Pleuraerguss muss mit der Entstehung eines Reexpansionsödems - auch bei langsamer Reexpansion der Lunge - gerechnet werden. In Anlehnung an

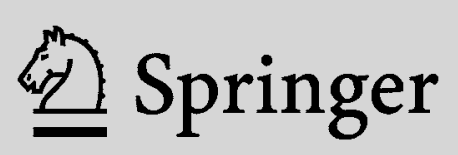


Chirurg 2005 $\cdot 76: 270-272$

DOI 10.1007/s00104-004-0985-7

○) Springer Medizin Verlag 2005

B. Hoksch - T. Weber · M. Beshay · R. Stein J. Schardt · R. A. Schmid

\section{Differentialdiagnose des pulmonalen Infiltrates nach Pneumothorax-Drainage}

\section{Zusammenfassung}

Das Reexpansionsödem der Lunge stellt insgesamt eine seltene Komplikation sowohl im Rahmen der Pneumothoraxtherapie als auch bei der Behandlung des ausgedehnten Pleuraergusses dar. Die Pathogenese ist nicht vollständig geklärt und scheint multifaktoriell zu sein. Bei Auftreten von Atembeschwerden nach Reexpansion einer zuvor kollabierten Lunge sollte unbedingt an ein Reexpansionsödem als mögliche Ursache gedacht werden.

\section{Schlüsselwörter}

Reexpansionsödem · Pneumothorax · Thoraxdrainage

\section{Differential diagnosis of pulmonary infiltration after insertion of a chest tube for pneumothorax}

\section{Abstract \\ Reexpansion injury of the lung is rare and may occur after reexpansion of a col- lapsed lung such as after evacuation of a large amount of air or fluid from the pleu- ral space. Its pathogenesis, however, is not clearly understood. Pulmonary ede- ma should always be considered if the pa- tient's respiratory condition worsens after lung reexpansion.}

\section{Keywords}

Reexpansion pulmonary edema .

Pneumothorax · Chest tube

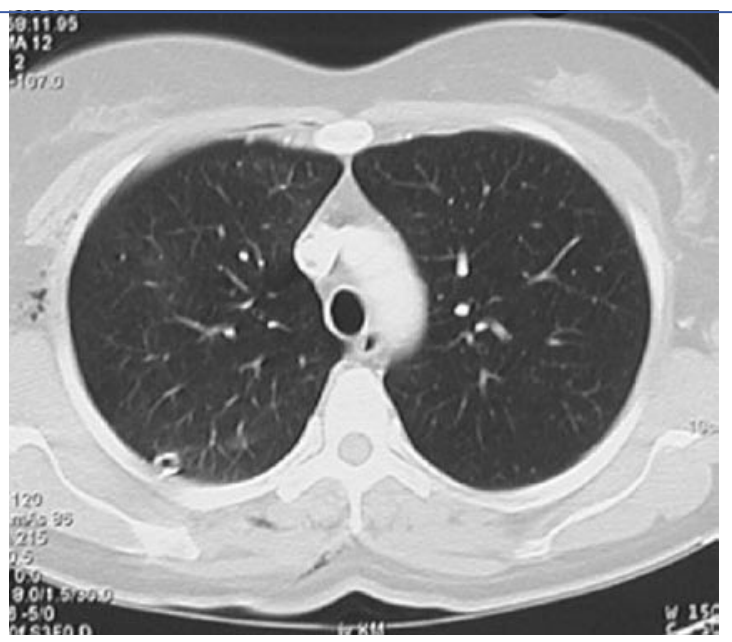

Abb. $3<$ Deutliche Regredienz des Ödems im Kontroll-CT

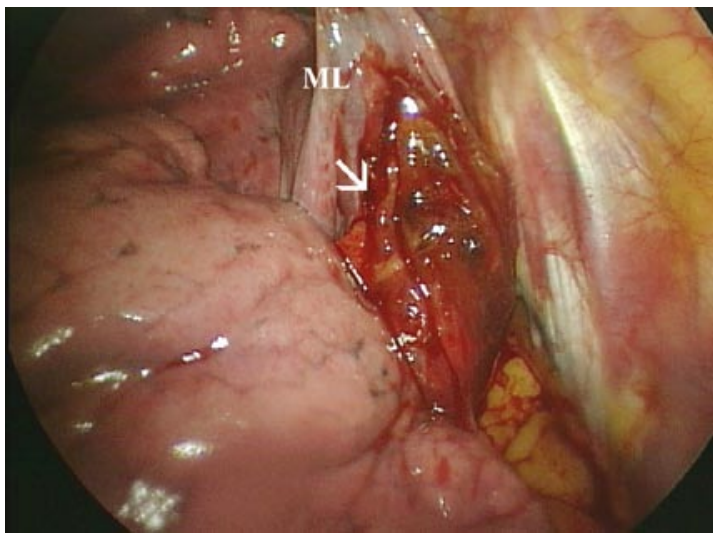

Abb. $4<$ Exposition der zu resezierenden eingebluteten Bulla (Pfeil) im Mittellappen (ML)

die Empfehlungen des American College of Chest Physicians (ACCP) von 2001 sollte daher bei einem ausgedehnten Pneumothorax ( $\geq 30 \%$ der Lunge) auf einen unmittelbaren Anschluss der Thoraxdrainage an einen Sog verzichtet werden [1]. Ob diese Technik im vorliegenden Fall die Ausbildung eines Reexpansionsödems verhindert hätte, bleibt allerdings ungewiss.

\section{Korrespondierender Autor Dr. B. Hoksch}

DMLL - Klinik für Thoraxchirurgie, Universitätsspital, $\mathrm{CH}-3010$ Bern E-Mail: beatrix.hoksch@insel.ch

Interessenkonflikt: Der korrespondierende Autor versichert, dass keine Verbindungen mit einer Firma, deren Produkt in dem Artikel genannt ist, oder einer Firma, die ein Konkurrenzprodukt vertreibt, bestehen.

\section{Literatur}

1. Baumann MH, Strange C, Heffner JE et al. (2001) Management of spontaneous pneumothorax - An American College of Chest Physicians Delphi Consensus Statement. Chest 119:590-602
2. Critchley LA, Au HK, Yim APC (1996) Reexpansion pulmonary edema occuring after thoracoscopic drainage of a pleural effusion. J Clin Anesth 8:591594

3. Fujino S, Tezuka N, Inoue $S$, Ichinose $M$, Hanaoka J, Sawai S, Kontani K (2000) Reexpansion pulmonary edema due to high-frequency jet ventilation: report of a case. Surg Today 30:1110-1111

4. Mahfood S, Hix WR, Aaron BL, Blaes P, Watson DC (1988) Reexpansion pulmonary edema. Ann Thorac Surg 45:340-345

5. Sakao Y, Kajikawa O, Martin TR, Nakahara Y, Hadden WA, Harmon CL, Miller EJ (2001) Association of IL-8 and MCP-1 with the development of reexpansion pulmonary edema in rabbits. Ann Thorac Surg 71:1825-1832

6. Scalzetti EM (2001) Unilateral pulmonary edema after talc pleurodesis. J Thorac Imag 16:99-102

7. Sherman SC (2003) Reexpansion pulmonary edema: a case report and review of the current literature. J Emerg Med 24:23-27

8. Sivrikoz MC, Tuncözgür B, Cekmen M et al. (2002) The role of tissue reperfusion in the reexppansion injury of the lungs. Eur J Cardio Thorac Surg 22:721-727

9. Suzuki S, Niikawa H, Shibuya J, Hosaka T, Maeda S, Suzuki T, Handa M (2002) Analysis of edema fluids and histologic features of the lung in reexpansion pulmonary edema during video-assisted thoracoscopic surgery. J Thorac Cardiovasc Surg 123:387389 